\title{
Data Classification with a Relaxed Model of Variable Kernel Density Estimation
}

\author{
Yen-Jen Oyang*, Yu-Yen Ou, Shien-Ching Hwang, \\ Chien-Yu Chen ${ }^{1}$, and Darby Tien-Hau Chang \\ Department of Computer Science and Information Engineering, \\ National Taiwan University, Taipei, Taiwan, R.O.C. \\ ${ }^{1}$ Graduate School of Biotechnology and Bioinformatics, \\ Yuan-Ze University, Chung-Li, 320, Taiwan, R.O.C.
}

yjoyang@csie.ntu.edu.tw; yien@csie.ntu.edu.tw; schwang@mars.csie.ntu.edu.tw

\begin{abstract}
In recent years, kernel density estimation has been exploited by computer scientists to model several important problems in machine learning, bioinformatics, and computer vision. However, in case the dimension of the data set is high, then the conventional kernel density estimators suffer poor convergence rates of the pointwise mean square error (MSE) and the integrated mean square error (IMSE). Therefore, design of a novel kernel density estimator that overcomes this problem has been a great challenge for many years. This paper proposes a relaxed model of the variable kernel density estimation and analyzes its performance in data classification applications. It is proved in this paper that, in terms of pointwise MSE, the convergence rate of the relaxed variable kernel density estimator can approach $O\left(n^{-1}\right)$ regardless of the dimension of the data set, where $n$ is the number of sampling instances. Experiments with the data classification applications have shown that the improved convergence rate of the pointwise MSE leads to higher prediction accuracy. In fact, the experimental results have also shown that the data classifier constructed based on the relaxed variable kernel density estimator is capable of delivering the same level of prediction accuracy as the SVM with the Gaussian kernel.
\end{abstract}

Keyterms: kernel density estimation, variable kernel density estimation, radial basis function, RBF network, data classification.

\section{INTRODUCTION}

Kernel density estimation is a problem that has been studied by statisticians for several decades $[1,5,10,22,23]$. In recent years, kernel density estimation has been exploited by computer scientists to model several important problems in machine learning [9], bioinformatics [6], and computer vision [3]. The problem definition of kernel density estimation is as follows: given a set of sampling instances $\left\{s_{1}\right.$, $\left.s_{2}, \ldots, s_{n}\right\}$ randomly taken from the target probability distribution with an unknown form in an $m$-dimensional vector space, we want to construct an estimator of the following form that provides a good approximation of the target probability density function:

$$
\hat{f}(v)=\sum_{i=1}^{n} w_{i} \phi\left(v ; s_{i}, \sigma_{i}\right), \text { where }
$$

1) $v$ is a vector in the $m$-dimensional vector space;

2) $w_{i}$ and $\sigma_{i}$ are the weight and width of the $i$-th kernel function, respectively.

So far, many types of kernel functions have been proposed for kernel density estimation. If the popular Gaussian function is employed, then the form of equation (1) becomes

$$
\hat{f}(v)=\sum_{i=1}^{n} w_{i} \exp \left(-\frac{\left\|v-s_{i}\right\|^{2}}{2 \sigma_{i}^{2}}\right),
$$

where $\left\|v-s_{i}\right\|$ is the distance between vectors $v$ and $s_{i}$.

There are several types of kernel density estimators proposed by statisticians [22]. The basic one is the fixed kernel density estimator, which is of the following form with the Gaussian function employed:

$$
\hat{f}(v)=\frac{1}{n} \cdot \frac{1}{(\sqrt{2 \pi} \sigma)^{m}} \sum_{i=1}^{n} \exp \left(-\frac{\left\|v-s_{i}\right\|^{2}}{2 \sigma^{2}}\right) .
$$

Since in a fixed kernel density estimator all kernel functions have the same width, it is of interest to investigate the effects of the generalized models.

One major approach that has been proposed to generalize the fixed kernel density estimator is the variable kernel density estimator [5]. The general form of the variable kernel density estimator with the Gaussian function is as follows:

$$
\hat{f}(v)=\frac{1}{n} \cdot \sum_{i=1}^{n} \frac{1}{\left(\sqrt{2 \pi} \theta R_{k}\left(s_{i}\right)\right)^{m}} \exp \left(-\frac{\left\|v-s_{i}\right\|^{2}}{2\left(\theta R_{k}\left(s_{i}\right)\right)^{2}}\right),
$$

where $\theta$ is the so-called smoothing parameter and $R_{k}\left(s_{i}\right)$ is the distance between sampling instance $s_{i}$ and its $k$-th nearest neighbor. As equation (4) shows, in a variable kernel

\footnotetext{
- To whom correspondence should be addressed.

This research was sponsored by National Science Council of R.O.C. under contract NSC 92-2213-E-002-095.
} 
density estimator, the width of each kernel function is a function of the local density surrounding the sampling instance. The motivation behind the development of the variable kernel density estimator is to exploit the local distribution surrounding each sampling instance. In this regard, the adaptive kernel density estimator represents another major alternative approach to generalize the fixed kernel density estimator $[1,22]$.

The main problem with the conventional kernel density estimators in handling high dimensional data sets is that the convergence rate of the pointwise MSE(mean square error) turns poor as the dimension increases. For example, with the Gaussian function, the convergence rate of the fixed kernel density estimator is $O\left(n^{-4 /(m+4)}\right)$, where $m$ is the dimension of the data set [22]. With some specific types of kernel functions, the convergence rate can be slightly improved to $O\left(n^{-8 /(m+8)}\right)$. Nevertheless, regardless of the type of the kernel functions employed, the pointwise MSE of a conventional kernel density estimator converges at an extremely slow rate in case the dimension of the data set is high.

One important development in recent years toward the design of a multivariate kernel density estimator that features a higher convergence rate of the pointwise MSE is due to Sain and Scott [21]. In the article, Sain and Scott showed that it is feasible to make the pointwise MSE of the socalled locally adaptive kernel density estimator converge at $O\left(n^{-1}\right)$ in regions where the target probability density function is convex. Nevertheless, their theorems hold only in such regions.

This paper proposes a relaxed model of the variable kernel density estimation. The most important mathematical property of the relaxed model is that the convergence rate of the pointwise MSE can approach $O\left(n^{-1}\right)$, regardless of the dimension of the data set. In fact, the development of the relaxed variable kernel density estimation stems from our recent work with the $\mathrm{RBF}$ (radial basis function) network $[18,19]$. The experimental results reported in our recent article [19] show that the proposed learning algorithm for the RBF network, which is based on a preliminary version of the relaxed variable kernel density estimator, is capable of delivering the same level of accuracy as the SVM(support vector machine) $[7,11]$ in data classification applications. On the other hand, it generally takes far less time than the SVM algorithm for constructing a data classifier with an optimized parameter setting, due to its average time complexity of $O(n \log n)$. Therefore, it is of interest to investigate the properties of the relaxed model of variable kernel density estimation from a theoretical aspect.

In the mathematical analysis presented in this paper, the discussion will focus on how the pointwise MSE of a kernel density estimator behaves, because the application addressed in this paper is data classification. With respect to data classification accuracy, the main concern is the point- wise MSE in the critical region, instead of the IMSE(integrated MSE) [22] over the entire distribution.

In the following part of this paper, section II discusses the works related to the study presented in this paper. Section III elaborates the mathematical properties of the relaxed variable kernel density estimator. Section IV reports the experiments conducted to evaluate its performance in data classification applications and discusses interesting observations. Finally, concluding remarks are presented in section V.

\section{RELATED WORKS}

The general form of a kernel density estimator shown in equation (1) is in fact the same as that of an output node of an RBF network. In the machine learning research community, there have been quite a few learning algorithms proposed for RBF networks. The learning algorithm determines the number of nodes in the hidden layer, the activation functions associated with the hidden nodes, and the weights associated with the links between the hidden and output layers.

Basically, there are two categories of algorithms proposed for constructing RBF networks [2, 16]. The first category of algorithms simply places one radial basis function at each sampling instance [17] and yields the same mathematical form for each output node as equation (1). The kernel density estimation based algorithms proposed in recent years represent the latest developments in the design of this category of algorithms $[8,9]$. One desirable feature of the kernel density estimation based approach is its low time complexity of $O(n \log n)$ for construction of a classifier. However, because the RBF network constructed with this category of algorithms is instance based, data reduction may need to be invoked to reduce the size of the RBF network constructed. Another problem with this approach is that the conventional kernel density estimation algorithms can not deliver satisfactory accuracy in handling high dimensional data sets. In this regard, our recent studies have shown that the RBF network constructed with the earlier version of the relaxed model of variable kernel density estimation is capable of delivering the same level of accuracy as the SVM in data classification applications $[18,19]$.

The second category of algorithms proposed for constructing RBF networks resorts to a regularization process to figure out the optimal parameter settings of the RBF network $[12,13,14,15]$. Because the regularization process involves computing the inverse of a matrix with dimension equal to the number of hidden nodes in the network, it is typical that a clustering analysis on the training data set is conducted to determine where the radial basis functions should be located $[2,12,14]$. One of the main advantages of this approach is that the size of the RBF network con- 
structed is relatively small. A comprehensive analysis on the characteristics of the RBF network constructed with this category of algorithms can be found in one of our latest articles $[20]$.

In summary, kernel density estimation and regularization represent two most recent approaches proposed for construction of RBF networks. Both approaches offer some advantages and suffer some deficiencies. Nevertheless, the experimental results presented in this article and in another our latest article show that with the state-of-art algorithms both approaches can deliver the same level of accuracy in data classification applications [20]. Therefore, it is really up to the user to select the approach that is most suitable based on the characteristics of the data set being handled.

\section{The RelaXed Model OF VARIABLE KeRnel DENSITY ESTIMATION}

Let $\left\{s_{1}, s_{2}, \ldots, s_{n}\right\}$ be a set of sampling instances independently and randomly taken from a distribution with probability density function $f$ in an $m$-dimensional vector space. The general form of the relaxed variable kernel density estimator is as follows:

$$
\hat{f}(v)=\frac{1}{n} \cdot \sum_{i=1}^{n} \frac{1}{\left(\sqrt{2 \pi} \sigma_{i}\right)^{\alpha}} \exp \left(-\frac{\left\|v-s_{i}\right\|^{2}}{2 \sigma_{i}^{2}}\right),
$$

where

1) $\sigma_{i}=\beta \cdot\left[n \ddot{f}\left(s_{i}\right)\right]^{-1 / \alpha}$;

2) $\alpha$ is a positive real number;

3) $\beta$ is the smoothing parameter;

4) $\ddot{f}\left(s_{i}\right)$ is a pilot estimate of $f\left(s_{i}\right)$.

In our implementation, the nearest neighbor estimator [19, 22] shown in the following has been adopted to obtain a pilot estimate of $f\left(s_{i}\right)$ :

$$
\ddot{f}\left(s_{i}\right)=\frac{(k+1)}{n}\left[\frac{\left(R_{k}\left(s_{i}\right) \sqrt{\pi}\right)^{m}}{\Gamma\left(\frac{m}{2}+1\right)}\right]^{-1} \cong f\left(s_{i}\right),
$$

where $R_{k}\left(s_{i}\right)$ is the distance between sampling instance $s_{i}$ and its $k$-th nearest neighbor.

In the following discussion, without loss of generality, we can assume that it is the pointwise MSE at the origin that is of our concern. In addition, we will set the smoothing parameter $\beta$ as follows:

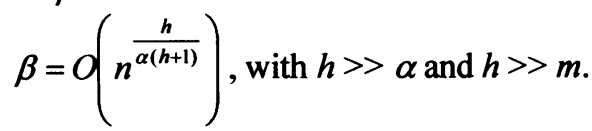

With such setting, we have $\frac{\beta}{n^{1 / \alpha}} \rightarrow 0$ as $n \rightarrow \infty$.

The first theorem that we will prove is that, with proper setting of parameter $\alpha$, we can make the relaxed variable kernel density estimator shown in equation (5) unbiased, provided that $n$ is sufficiently large.
Theorem 1. Let $\hat{f}(v)$ be a relaxed variable kernel density estimator of $f(v)$ as shown in equation (5). Then, when $n$ is sufficiently large, there exists a real number $\hat{\alpha}$ within $(m-1, m+1)$ that makes $E[\hat{f}(0)]=f(0)$, provided that $0<f(0)<\infty$.

Proof. A complete proof is presented in Appendix A.

In Theorem 2, we will derive a closed form of the variance of the relaxed variable kernel density estimator.

Theorem 2. Let $\hat{f}(v)$ be a relaxed variable kernel density estimator of $f(v)$. Then, when $n$ is sufficiently large,

$$
\operatorname{Var}[\hat{f}(\mathbf{0})] \cong \frac{\pi^{m / 2} f(\mathbf{0}) \ddot{f}(\mathbf{0})^{2-m / \alpha}}{(2 \pi)^{\alpha} n^{m / \alpha-1} \beta^{2 \alpha-m}} .
$$

Proof. A complete proof is presented in Appendix B.

Theorem 3. Assume that the smoothing parameter $\beta$ in equation (5) is set to $O\left(n^{(h / \alpha(h+1))}\right)$ with $h>>\alpha$ and $h>m$. Then, when $n$ is sufficiently large, there exists a real number $\hat{\alpha}$ within $(m-1, m+1)$ that makes

$$
\operatorname{MSE}[\hat{f}(0)] \cong O\left(n^{-1+\eta}\right) \text {, where } \eta \rightarrow 0 \text { as } h \rightarrow \infty .
$$

Proof.

By definition, $\operatorname{MSE}[\hat{f}(\mathbf{0})]=\operatorname{VAR}[\hat{f}(\mathbf{0})]+[E[\hat{f}(\mathbf{0})]-f(\mathbf{0})]^{2}$.

According to Theorems 1 and 2 , when $n$ is sufficiently large, we can find an $\hat{\alpha}$ within $(m-1, m+1)$ that makes

$$
\begin{aligned}
& \operatorname{MSE}[\hat{f}(\mathbf{0})]=\left\{V A R[\hat{f}(\mathbf{0})]+[E[\hat{f}(\mathbf{0})]-f(\mathbf{0})]^{2}\right\} \\
& \cong O\left(\frac{1}{n^{m / \hat{\alpha}-1} \cdot \beta^{2 \hat{\alpha}-m}}\right)=O\left(n^{\frac{\hat{\alpha}-m-\hat{\alpha} h}{\hat{\alpha}(h+1)}}\right)=O\left(n^{-1+\eta}\right),
\end{aligned}
$$

where $\eta \rightarrow 0$ as $h \rightarrow \infty$.

So far, we have shown that we can make the convergence rate of the pointwise MSE of the relaxed variable kernel density estimator presented in equation (5) approach $O\left(n^{-1}\right)$, regardless of the dimension of the dataset, provided that $n$ is sufficiently large. However, in case $n$ is not large enough, then $\hat{\alpha}$ in Theorems 1 and 3 may fall in a wider range beyond $(m-1, m+1)$.

\section{IMPLEMENTATION AND EXPERIMENTAL RESULTS}

This section addresses the implementation of a supervised learning algorithm based on the relaxed variable kernel density estimator presented in Section III and reports the experiments conducted to evaluate its performance. It is assumed that the sampling instances in the data set are distributed in an $m$-dimensional vector space. The learning algorithm will construct one kernel density estimator based on equations (5) and (6) for approximating the distribution of 
one class of sampling instances in the vector space. Then, a query instance located at $v$ is predicted to belong to the class that gives the maximum value of the likelihood functions defined in the following:

$$
L_{j}(v)=\frac{\left|S_{j}\right|}{|S|} \hat{f}_{j}(v),
$$

where $S_{j}$ is the set of class- $j$ training instances, $S$ is the set of training instances of all classes, and $\hat{f}_{j}(v)$ is the kernel density estimator of class- $j$ training instances. In our implementation, we have observed that $R\left(s_{i}\right)$ in equation (6) is determined by one single training instance and therefore could be unreliable, if the data set is noisy. Accordingly, we have adopted $\bar{R}\left(s_{i}\right)$ defined in the following to replace $R\left(s_{i}\right)$ in equation (6):

$$
\bar{R}\left(s_{i}\right)=\frac{m+1}{m}\left(\frac{1}{k} \sum_{h=1}^{k}\left\|\hat{s}_{h}-s_{i}\right\|\right),
$$

where $\hat{\boldsymbol{s}}_{1}, \hat{\boldsymbol{s}}_{2}, \ldots, \hat{\boldsymbol{s}}_{\boldsymbol{k}}$ are the $k$ nearest training instances of the same class as $s_{i}$. The basis of employing $\bar{R}\left(s_{i}\right)$ can be found in [19]. As far as the time complexity of the learning algorithm is concerned, it has been shown in [19] that the average time complexity for constructing a data classifier based on the relaxed variable kernel density estimator is bounded by $O(n \log n)$, where $n$ is total number of training instances. Furthermore, the average time complexity for predicting the classes of $n^{\prime}$ query instances is bounded by $O\left(n^{\prime} \log n\right)$.

In the remaining part of this section, we will reports the experiments conducted to evaluate the performance of the data classifier constructed based on the relaxed variable kernel density estimator. In the experiments, we have employed both synthesized datasets and some of benchmark datasets in the UCI repository [4]. The experiments with the synthesized datasets have been designed to pin down the main characteristics of the data classifier. On the other hand, the experiments with the UCI benchmark datasets are aimed at studying how the data classifier performs in handling real data sets.

TABLE 1(a) shows the underlying probability distributions of the synthesized datasets, from which the training and testing instances are randomly taken with the total number of negative instances equal to 20 times the total number of positive instances. The design of the synthesized data sets is aimed at emulating skewed data sets with very few positive instances. In the experiments, we focus on the distributions of instances in the proximity of the origin. In this region, the number of positive instances is slightly larger than the number of negative instances. In our study, we have investigated the effects of replace $m$ in equations (6) and (7) by $\alpha$, which implies that we have treated the data set as if it were in an $\alpha$-dimensional vector space. Since the results have shown that replacing $m$ by $\alpha$ generally leadeds to higher classification accuracy, in the following discussion we will only report the experimental results obtained with this practice adopted.

TABLE 1(b) and TABLE 1(c) show the summarized results with 20 independent runs of the same experiment procedure. For each run of the experiment reported in TABLE 1(b), a total of $10,000,000$ training instainces along with $2,500,000$ testing instances have been independently generated and only the instances that fall within 0.2 away from the origin have been included in the data set. For each run of the experiment reported in TABLE 1(c), a total of $20,000,000$ training instances along with 5,000,000 testing instances have been independently generated and only the instances that fall within the same sphere have been included in the data set. Then, the relaxed variable kernel density estimator based data classifiers constructed with the training instances under various parameter settings have been used to predict the classes of the testing instances. In these experiments, parameter $k$ in equations (6) and (7) has been set to 30 and the maximum prediction accuracies obtained with the following possible combinations of $\alpha$ and $\beta$ have been reported in TABLE 1(b) and TABLE 1(c):

$$
\{\alpha \mid \alpha=1.0,1.1,1.2, \ldots, 4.0\} \times\{\beta \mid \beta=0.5,0.6,0.7, \ldots, 6.0\} .
$$

Since the underlying probability distributions are in a 3dimensional vector space, when $\alpha$ is set to 3 , equation (5) in fact becomes the conventional variable kernel density estimator. According to the results summarized in TABLE 1(b) and TABLE 1(c), the confidence levels for accepting that the data classifier based on the relaxed variable kernel density estimator is capable of delivering higher prediction accuracy than the data classifier based on the conventional variable kernel density estimator are over $\mathbf{9 0 \%}$ and $95 \%$, respectively.

TABLE 1. EXPERIMENTAL RESULTS WITH THE SYNTHESIZED DATASETS.

\begin{tabular}{|l|l|}
\hline $\begin{array}{l}\text { Probability density } \\
\text { function of the posi- } \\
\text { tive instances }\end{array}$ & $\frac{1}{(\sqrt{2 \pi})^{3}} \exp \left(-\frac{1}{2}\left(x^{2}+y^{2}+z^{2}\right)\right)$ \\
\hline $\begin{array}{l}\text { Probability density } \\
\text { function of the nega- } \\
\text { tive instances }\end{array}$ & $\frac{1}{(\sqrt{2 \pi})^{3}} \exp \left[\frac{-1}{2}\left[(x-2.5)^{2}+y^{2}+z^{2}\right]\right]$ \\
\hline
\end{tabular}

(a) The underlying probability distributions

\begin{tabular}{|l|l|l|l|l|}
\hline $\begin{array}{l}\text { Avg. \# of } \\
\text { training } \\
\text { instances }\end{array}$ & $\begin{array}{l}\text { Avg. \# of } \\
\text { testing } \\
\text { instances }\end{array}$ & $\begin{array}{l}\text { Accuracy } \\
\alpha=1.3, \beta=1.6 \\
(\mu \pm \sigma)\end{array}$ & $\begin{array}{l}\text { Accuracy } \\
\alpha=3, \beta=0.8 \\
(\mu \pm \sigma)\end{array}$ & $\begin{array}{l}\text { F } \\
\text { statistic }\end{array}$ \\
\hline 3629.6 & 921.95 & $\begin{array}{l}52.32 \% \\
\pm 1.91 \%\end{array}$ & $\begin{array}{l}51.30 \% \\
\pm 1.62 \%\end{array}$ & $\begin{array}{l}3.45> \\
F_{0.1}\end{array}$ \\
\hline
\end{tabular}

(b) Summarized experimental result with the first data set.

\begin{tabular}{|l|l|l|l|l|}
\hline $\begin{array}{l}\text { Avg. \# of } \\
\text { training } \\
\text { instances }\end{array}$ & $\begin{array}{l}\text { Avg. \# of } \\
\text { testing } \\
\text { instances }\end{array}$ & $\begin{array}{l}\text { Accuracy } \\
\alpha=1.0, \beta=2.9 \\
(\mu \pm \sigma)\end{array}$ & $\begin{array}{l}\text { Accuracy } \\
\alpha=3, \beta=0.8 \\
(\mu \pm \sigma)\end{array}$ & $\begin{array}{l}\text { F } \\
\text { statistic }\end{array}$ \\
\hline 7271.2 & 1810.45 & $\begin{array}{l}52.84 \% \\
\pm 1.30 \%\end{array}$ & $\begin{array}{l}51.97 \% \\
\pm 1.30 \%\end{array}$ & $\begin{array}{l}4.52> \\
F_{0.05}\end{array}$ \\
\hline
\end{tabular}

(c) Summarized experimental result with the second data set. 
TABLE 2. COMPARISON OF THE PREDICTION ACCURACY OF ALTERNATIVE APPROACHES WITH SEVEN BENCHMARK DATASETS IN THE UCI REPOSITORY [4]

\begin{tabular}{|l|c|c|c|c|c|c|c|c|}
\hline & iris & wine & Vowel & segment & satimage & letter & shuttle & Average \\
\hline Relaxed variable KDE & 96.67 & 99.44 & 99.62 & 97.403 & 92.45 & 97.12 & 99.94 & 97.52 \\
\hline Parameter $\alpha$ in equation (5) & 4 & 1 & 1 & 1.4 & 2.1 & 2 & 3 & - \\
\hline Conventional variable KDE & 96.67 & 95.52 & 99.62 & 97.27 & 89.35 & 96.68 & 99.94 & 96.44 \\
\hline Dimension of the data set & 4 & 13 & 10 & 19 & 36 & 16 & 9 & - \\
\hline SVM (Guassian kernel) & 97.33 & 99.44 & 99.05 & 97.40 & 91.30 & 97.98 & 99.92 & 97.49 \\
\hline SVM (Linear kernel) & 97.33 & 98.88 & 81.82 & 95.80 & 85.85 & 85.14 & 98.10 & 91.85 \\
\hline
\end{tabular}

TABLE 2 shows how the data classifier based on the relaxed variable kernel density estimator performs, in terms of prediction accuracy, in comparison with the alternative approaches with 7 benchmark data sets from the UCI repository. The experimental procedure employed in this experiment is exactly the same as that employed in our recent article [19]. In addition, the implementation of the relaxed variable kernel density estimator based classifier is essentially the same as the implementation detailed in our recent article [19]. The only difference between these two implementations is that $\alpha$ in equation (5) can be set to any positive real number in the current implementation, instead of only to a positive integer in our previous implementation. In these experiments, parameter $\beta$ in equation (5) has been set to the default value, which is 0.7 .

As the data in TABLE 2 reveals, the data classifier based on the relaxed variable kernel density estimator is capable of delivering the same level of classification accuracy as the LIBSVM package with the Gaussian kernel [11] and the accuracy is generally higher than that delivered by the data classifier based on the conventional variable kernel density estimator. The classification accuracy of the SVM with the linear kernel is also provided as reference.

\section{CONCLUSION}

This paper proposes a relaxed model of variable kernel density estimation and studies how it performs in data classification applications. The most important feature of the relaxed model is that we can make the convergence rate of the pointwise MSE approach $O\left(n^{-1}\right)$, regardless of the dimension of the data set. In the benchmark experiments reported in this paper, the data classifier based on the relaxed variable kernel density estimator has been able to deliver the same level of classification accuracy as the SVM with the Gaussian kernel and has performed generally better than the data classifier based on the conventional variable kernel density estimator. Another important feature of the relaxed variable kernel density estimation based approach is its low time complexity for construction of a data classifier, on average $O(n \log n)$, where $n$ is the number of training instances.

As the experimental results reported in this paper are quite promising, it is of great interest to investigate how the data classifier constructed based on the relaxed variable kernel density estimator performs in emerging bioinformatics applications, in which data sets with extremely high dimensions, e.g. hundreds or thousands of dimensions, are common. Another interesting issue is how the relaxed variable kernel density estimator can be exploited in other machine learning problems, e.g. data clustering.

\section{APPENDIX A}

Proof of Theorem 1.

Let $z=\left(x_{1}, x_{2}, \ldots, x_{m}\right)$ denote the coordinate of one single sampling instance, then we have

$E[\hat{f}(0)]=$

$n \int_{-\infty}^{\infty} \cdots \int_{-\infty}^{\infty} \int_{-\infty}^{\infty} \frac{f(z)}{n} \cdot \frac{\exp \left(-\|z\|^{2} / 2 \sigma^{2}(z)\right)}{[\sqrt{2 \pi} \sigma(z)]^{\alpha}} d x_{1} d x_{2} \cdots d x_{m}$.

Since $\frac{\beta}{n^{1 / \alpha}} \rightarrow 0$ as $n \rightarrow \infty$, we have

$\sigma(z)=\beta \cdot[n \ddot{f}(z)]^{-1 / \alpha} \rightarrow 0$ and

$\frac{\exp \left(-\|z\|^{2} / 2 \sigma^{2}(z)\right)}{[\sqrt{2 \pi} \sigma(z)]^{m}}$ approaching a multi-dimensional

Dirac delta function. Therefore, as $n \rightarrow \infty$, we have

$E[\hat{f}(\mathbf{0})] \rightarrow \int_{-\infty}^{\infty} \cdots \int_{-\infty}^{\infty} \int_{-\infty}^{\infty} f(z)[\sqrt{2 \pi} \sigma(z)]^{m-\alpha} \delta(z) d x_{1} d x_{2} \cdots d x_{m}$

$=n^{1-\frac{m}{\alpha}} \cdot(\sqrt{2 \pi} \beta)^{m-\alpha} \cdot f(\mathbf{0}) \cdot[\ddot{f}(\mathbf{0})]^{1-\frac{m}{\alpha}}$,

where $\delta(z)$ is the multi-dimensional Dirac delta function.

Accordingly, if we set $\alpha=m-1$, then as $n \rightarrow \infty$

$$
E[\hat{f}(0)] \rightarrow 0<f(0) \text { due to } \frac{\beta}{n^{1 / \alpha}} \rightarrow 0 .
$$

On the other hand, if we set $\alpha=m+1$, then as $n \rightarrow \infty$

$$
E[\hat{f}(\mathbf{0})] \rightarrow \infty>f(\mathbf{0}) .
$$

Since $E[\hat{f}(0)]$ is a continuous function of $\alpha$ for $\alpha>0$, when $n$ is sufficiently large, there exists a real number $\hat{\alpha}$ within $(m-1, m+1)$ that makes $E[\hat{f}(\mathbf{0})]=f(0)$.

\section{APPENDIX B}

Proof of Theorem 2.

Let $z=\left(x_{1}, x_{2}, \ldots, x_{m}\right)$ denote the coordinate of sampling instance $s_{i}$ and 
$\hat{f}_{1 / n}(0)=\frac{1}{n} \cdot \frac{\exp \left(-\|z\|^{2} / 2 \sigma^{2}(z)\right)}{[\sqrt{2 \pi} \sigma(z)]^{\alpha}}$.

Then, as $n \rightarrow \infty$ we have

$E\left[\hat{f}_{1 / n}^{2}(0)\right]=\int_{-\infty} \int_{-\infty} \cdots \int_{-\infty}^{\infty} f(z) \cdot \frac{1}{n^{2}} \cdot \frac{\exp \left(-\|z\|^{2} / \sigma^{2}(z)\right)}{[\sqrt{2 \pi} \sigma(z)]^{2 \alpha}} d x_{1} d x_{2} \cdots d x_{m}$

$=\frac{1}{n^{2}} \cdot \int_{-\infty}^{\infty} \int_{-\infty}^{\infty} \cdots \int_{-\infty}^{\infty} \frac{f(z)[\sqrt{\pi} \sigma(z)]^{m}}{[\sqrt{2 \pi} \sigma(z)]^{2 \alpha}} \cdot \frac{\exp \left(-|z|^{2} / \sigma^{2}(z)\right)}{[\sqrt{\pi} \sigma(z)]^{m}} d x_{1} d x_{2} \cdots d x_{m}$

$\rightarrow \frac{\pi^{m / 2} f(0) \ddot{f}(0)^{2-m / \alpha}}{(2 \pi)^{\alpha} n^{m / \alpha} \beta^{2 \alpha-m}}$,

since $\frac{\exp \left(-|z|^{2} / \sigma^{2}(z)\right)}{[\sqrt{\pi} \sigma(z)]^{m}}$ approaches a multi-dimensional Dirac

delta function. Concerning $\operatorname{Var}\left[\hat{f}_{1 / n}(0)\right]$, we have

$$
\begin{aligned}
& E\left[\hat{f}_{1 / n}^{2}(\mathbf{0})\right] \rightarrow \mathcal{Q}\left(\frac{1}{n \beta^{\alpha}} \frac{\beta^{m-\alpha}}{n^{m / \alpha-1}}\right) \text { and } \\
& E^{2}\left[\hat{f}_{1 / n}(\mathbf{0})\right]=\left(\frac{1}{n} E[\hat{f}(\mathbf{0})]\right)^{2} \rightarrow O\left(\left[\frac{1}{n} \cdot \frac{\beta^{m-\alpha}}{n^{m / \alpha-1}}\right]^{2}\right) \text { as } n \rightarrow \infty .
\end{aligned}
$$

Therefore, as $n \rightarrow \infty$, we have

$$
\operatorname{Var}\left[\hat{f}_{1 / n}(0)\right] \rightarrow E\left[\hat{f}_{1 / n}^{2}(0)\right] \text { due to } \frac{\beta^{\alpha}}{n} \rightarrow 0 \text {. }
$$

Furthermore, based on the assumption that all the sampling instances are randomly and independently taken from $f$, when $n$ is sufficiently large, we have

$$
\begin{aligned}
& \operatorname{Var}[\hat{f}(\mathbf{0})]=n \cdot \operatorname{Var}\left[\hat{f}_{1 / n}(\mathbf{0})\right] \cong n \cdot E\left[\hat{f}_{1 / n}^{2}(\mathbf{0})\right] \\
& =\frac{\pi^{m / 2} f(\mathbf{0}) \ddot{f}(\mathbf{0})^{2-m / \alpha}}{(2 \pi)^{\alpha} n^{m / \alpha-1} \beta^{2 \alpha-m}} .
\end{aligned}
$$

\section{References}

[1] I. S. Abramson, "On Bandwidth Variation in Kernel Estimates - A Square Root Law," The Annals of Statistics, vol. 10, no. 4, pp. 1217-1223, 1982.

[2] C. M. Bishop, "Improving the generalization properties of radial basis function neural networks," Neural Computation, vol. 3, no. 4, pp. 579-5881, 1991.

[3] M. J. Black, D. J. Fleet, and Y. Yacoob, "Robustly estimating changes in image appearance," Computer Vision and Image Understanding, vol. 78, no. 1, pp. 8-31, 2000.

[4] C. L. Blake and C. J. Merz, "UCI repository of machine learning databases," Technical report, University of California, Department of Information and Computer Science, Irvine, CA, 1998.

[5] L. Breiman, W. Meisel, and E. Purcell, "Variable kernel estimates of multivariate densities," Technometrics, vol. 19, pp. 135-144, 1977.

[6] D. Ti.-H. Chang, C.-Y. Chen, W.-C. Chung, Y.-J. Oyang, H.F. Juan, and H.-C. Huang, "ProteMiner-SSM: A Web Server for Identifying Possible Protein-Ligand Interactions Based on Analysis of Protein Tertiary Substructures," Nucleic Acids Research, vol. 32 (Web Server issue), W76-W82, 2004.

[7] C. Cortes and V. Vapnik. "Support-vector network," Machine Learning, vol. 20, pp. 273-297, 1995.
[8] M. N. Dailey, G. W. Cottrell, and T. A. Busey, "Facial memory is kernel density estimation (almost)," Advances in Neural Information Processing Systems, vol. 11, pp. 24-30, 1998.

[9] G. L. David, "Similarity metric learning for a variable-kernel classifier," Neural Computation, vol. 7, no. 1, pp. 72-85, 1995.

[10] L. Devroye. A Course in Density Estimation. In Birkhauser:Boston MA, 1987.

[11] C. W. Hsu and C. J. Lin. "A comparison of methods for multi-class support vector machines," IEEE Transactions on Neural Networks, vol. 13, no. 2, pp. 415-425, 2002.

[12] Y. S. Hwang and S. Y. Bang, "An efficient method to construct a radial basis function neural network classifier," Neural Networks, vol. 10, no. 8, pp. 1495-1503, 1997.

[13] J. Moody and C. J. Darken, "Fast learning in networks of locally-tuned processing units," Neural Computation, vol. 1, no. 2, pp. 281-294, 1989.

[14] M. Musavi, W. Ahmed, K. Chan, K. Faris, and D. Hummels, "On the training of radial basis function classifiers," Neural Networks, vol. 5, no. 4, pp. 595-603, 1992.

[15] M. J. L. Orr, "Regularisation in the selection of radial basis function centres," Neural Computation, vol. 7, no. 3, pp. 606623, 1995.

[16] M. J. L. Orr, "Introduction to radial basis function networks," Technical report, Center for Cognitive Science, University of Edinburgh, 1996.

[17] M. J. Orr, "Optimising the widths of radial basis function," Proceedings of the Fifth Brazilian Symposium on Neural Networks, pp. 26-29, 1998.

[18] Y.-J. Oyang, S.-C. Hwang, Y.-Y. Ou, C.-Y. Chen, and Z.-W. Chen, "A Novel Learning Algorithm for Data Classification with Radial Basis Function Networks," Proceedings of 9th International Conference on Neural Information Processing, pp. 1021-1026, 2002.

[19] Y.-J. Oyang, S.-C. Hwang, Y.-Y. Ou, C.-Y. Chen, and Z.-W. Chen, "Data Classification with Radial Basis Function Networks Based on a Novel Kernel Density Estimation Algorithm", IEEE Transactions on Neural Networks, vol. 16, no. 1, pp. 225-236, 2005.

[20] Y.-Y. Ou, C.-Y. Chen, Y.-J. Oyang, "Data Classification Based on Radial Basis Function Networks Constructed with An Incremental Hierarchical Clustering Algorithm", To appear in Proceedings of International Joint Conference on Neural Networks, 2005.

[21] S. R. Sain and D. W. Scott, "Zero-Bias Locally Adaptive Density Estimators," Scandinavian Journal of Statistics, vol. 29, no. 3, pp. 441, 2002.

[22] B. W. Silverman, Density Estimation for Statistics and Data Analysis, Chapman and Hall, London, 1986.

[23] G. R. Terrell and D. W. Scott, "Variable kernel density estimation," The Annals of Statistics, no. 20, pp. 1236-1265, 1992. 